\title{
Bavrin's Type Factorization of the Temljakov Operator for Holomorphic Functions in Circular Domains of $\mathbb{C}^{n}$
}

\author{
Renata Długosz ${ }^{1,2}$ - Piotr Liczberski ${ }^{2}$. \\ Edyta Trybucka ${ }^{3}$
}

Received: 11 July 2017 / Accepted: 18 January 2018 / Published online: 20 March 2018 (C) The Author(s) 2018

\begin{abstract}
The paper concerns investigations of holomorphic functions of several complex variables with a factorization of their Temljakov transform. Firstly, there were considered some inclusions between the families $\mathcal{C}_{\mathcal{G}}, \mathcal{M}_{\mathcal{G}}, \mathcal{N}_{\mathcal{G}}, \mathcal{R}_{\mathcal{G}}, \mathcal{V}_{\mathcal{G}}$ of such holomorphic functions on complete $n$-circular domain $\mathcal{G}$ of $\mathbb{C}^{n}$ in some papers of Bavrin, Fukui, Higuchi, Michiwaki. A motivation of our investigations is a condensation of the mentioned inclusions by some new families of Bavrin's type. Hence we consider some families $\mathcal{K}_{\mathcal{G}}^{k}, k \geq 2$, of holomorphic functions $f: \mathcal{G} \rightarrow \mathbb{C}, f(0)=1$, defined also by a factorization of $\mathcal{L} f$ onto factors from $\mathcal{C}_{\mathcal{G}}$ and $\mathcal{M}_{\mathcal{G}}$. We present some interesting properties and extremal problems on $\mathcal{K}_{\mathcal{G}}^{k}$.
\end{abstract}

Keywords Holomorphic functions on $n$-circular domains in $\mathbb{C}^{n}$. Minkowski function - Estimates of homogeneous polynomials of Taylor series - Temljakov operator · Bavrin's families of functions

Communicated by David Shoikhet.

$凶 \quad$ Piotr Liczberski

piotr.liczberski@p.lodz.pl

Renata Długosz

renata.dlugosz@p.lodz.pl

Edyta Trybucka

eles@ur.edu.pl

1 Centre of Mathematics and Physics, Lodz University of Technology, Al. Politechniki 11, 90-924 Lodz, Poland

2 Institute of Mathematics, Lodz University of Technology, Wólczańska 215, 93-005 Lodz, Poland

3 Faculty of Mathematics and Natural Sciences, University of Rzeszów, Prof. St. Pigonia 1, 35-310 Rzeszow, Poland 
Mathematics Subject Classification 32A30 - 30C45

\section{Introduction}

We say that a domain $\mathcal{G} \subset \mathbb{C}^{n}$, is complete $n$-circular if $z \lambda=\left(z_{1} \lambda_{1}, \ldots, z_{n} \lambda_{n}\right) \in \mathcal{G}$ for each $z=\left(z_{1}, \ldots, z_{n}\right) \in \mathcal{G}$ and every $\lambda=\left(\lambda_{1}, \ldots, \lambda_{n}\right) \in \overline{\mathcal{U}^{n}}$, where $\mathcal{U}$ is the unit $\operatorname{disc}\{\zeta \in \mathbb{C}:|\zeta|<1\}$. From now by $\mathcal{G}$ will be denoted a bounded complete $n$-circular domain in $\mathbb{C}^{n}, n \geq 2$. By $\mathcal{H}_{\mathcal{G}}$ let us denote the space of all holomorphic functions $f: \mathcal{G} \longrightarrow \mathbb{C}$ and by $\mathcal{H}_{\mathcal{G}}(1)$ the collection of all $f \in \mathcal{H}_{\mathcal{G}}$, normalized by $f(0)=1$.

Many authors (cf., eg., [1,2,5-7,11,18,19,23]) considered some Bavrin's subfamilies $\mathcal{X}_{\mathcal{G}}$ of the family $\mathcal{H}_{\mathcal{G}}(1)$. In the definitions of these families $\mathcal{X}_{\mathcal{G}}$ the main role play the families $\mathcal{C}_{\mathcal{G}}(\alpha), \alpha \in[0,1)$,

$$
\mathcal{C}_{\mathcal{G}}(\alpha)=\left\{f \in \mathcal{H}_{\mathcal{G}}(1): \operatorname{Re} f(z)>\alpha, z \in \mathcal{G}\right\}
$$

and the following invertible Temljakov [24] linear operator $\mathcal{L}: \mathcal{H}_{\mathcal{G}} \longrightarrow \mathcal{H}_{\mathcal{G}}$

$$
\mathcal{L} f(z)=f(z)+D f(z)(z), z \in \mathcal{G}
$$

where $D f(z)$ is the Fréchet derivative of $f$ at the point $z$. By a Bavrin's family $\mathcal{X}_{\mathcal{G}}$ we mean a collection of functions $f \in \mathcal{H}_{\mathcal{G}}(1)$ whose the Temljakov transform $\mathcal{L} f$ has a functional factorization $\mathcal{L} f=p \cdot g$, where $p \in \mathcal{C}_{\mathcal{G}} \equiv \mathcal{C}_{\mathcal{G}}(0)$ and $g$ is from a fixed subfamily of $\mathcal{H}_{\mathcal{G}}(1)$. Below, we recall the factorizations which define a few well known Bavrin's families $\mathcal{X}_{\mathcal{G}}$, like as

$$
\begin{aligned}
\mathcal{V}_{\mathcal{G}} & : \mathcal{L} f=p \cdot 1, p \in \mathcal{C}_{\mathcal{G}}, \\
\mathcal{M}_{\mathcal{G}} & : \mathcal{L} f=p \cdot f, p \in \mathcal{C}_{\mathcal{G}}, \\
\mathcal{N}_{\mathcal{G}} & : \mathcal{L} f=p \cdot \mathcal{L} \mathcal{L} f, p \in \mathcal{C}_{\mathcal{G}}, \\
\mathcal{R}_{\mathcal{G}} & : \mathcal{L} f=p \cdot \mathcal{L} \varphi, \varphi \in \mathcal{N}_{\mathcal{G}}, p \in \mathcal{C}_{\mathcal{G}} .
\end{aligned}
$$

It is known that functions of these families were used to construct biholomorphic mappings in $\mathbb{C}^{n}$ (cf., eg., [10,13,20]). Let us note that the above families have geometric interpretation, in particular the functions $f \in \mathcal{M}_{\mathcal{G}}$ map biholomorphically some planar intersections $\mathcal{S}$ of $\mathcal{G}$ onto starlike domains in $\mathbb{C}$,(see [1]). It is very important, because the starlikeness plays a central role in many different subjects of geometry and topology and in particular, in geometric function theory.

Let us recall also that Bavrin showed the inclusions $\mathcal{N}_{\mathcal{G}} \varsubsetneqq \mathcal{R}_{\mathcal{G}}, \mathcal{V}_{\mathcal{G}} \varsubsetneqq \mathcal{R}_{\mathcal{G}}$ and pointed that the first of them can be complete to the following double inclusion $\mathcal{N}_{\mathcal{G}} \varsubsetneqq$ $\mathcal{M}_{\mathcal{G}} \varsubsetneqq \mathcal{R}_{\mathcal{G}}$. Thus, it is natural to ask whether is possible to do the same in the case of the second above inclusion. In the paper [12] the authors defined a family $\mathcal{K}_{\mathcal{G}}^{-}$, which satisfies the inclusion $\mathcal{V}_{\mathcal{G}} \varsubsetneqq \mathcal{K}_{\mathcal{G}}^{-} \varsubsetneqq \mathcal{R}_{\mathcal{G}}$. An adequate definition of $\mathcal{K}_{\mathcal{G}}^{-}$has the form: A function $f \in \mathcal{H}_{\mathcal{G}}(1)$ belongs to $\mathcal{K}_{\mathcal{G}}^{-}$if its Temljakov transform $\mathcal{L} f$ has the factorization 


$$
\mathcal{L} f(z)=p(z) \cdot h(z) \cdot h(-z), z \in \mathcal{G}, h \in \mathcal{M}_{\mathcal{G}}\left(\frac{1}{2}\right), p \in \mathcal{C}_{\mathcal{G}}
$$

where the family $\mathcal{M}_{\mathcal{G}}(\alpha), \alpha \in[0,1)$, is defined similarly as $\mathcal{M}_{\mathcal{G}}$, but in this case $p \in \mathcal{C}_{\mathcal{G}}(\alpha)$.

In the present paper we consider Bavrin's type families $\mathcal{K}_{\mathcal{G}}^{k}, k \geq 2\left(\mathcal{K}_{\mathcal{G}}^{2}=\mathcal{K}_{\mathcal{G}}^{-}\right)$separating also the families $\mathcal{V}_{\mathcal{G}}, \mathcal{R}_{\mathcal{G}}$, i.e., satisfying the inclusions $\mathcal{V}_{\mathcal{G}} \varsubsetneqq \mathcal{K}_{\mathcal{G}}^{k} \varsubsetneqq \mathcal{R}_{\mathcal{G}}, k \geq 2$.

The formal definition of such family has the following form.

A function $f \in \mathcal{H}_{\mathcal{G}}(1)$ belongs to $\mathcal{K}_{\mathcal{G}}^{k}$ if there exist a function $p \in \mathcal{C}_{\mathcal{G}}$ and a function $h \in \mathcal{M}_{\mathcal{G}}\left(\frac{k-1}{k}\right)$ such that the Temljakov transform $\mathcal{L} f$ of $f$ has the factorization

$$
\mathcal{L} f(z)=p(z) \cdot \prod_{l=0}^{k-1} h\left(\varepsilon^{l} z\right), z \in \mathcal{G}
$$

where $\varepsilon=\varepsilon_{k}=\exp \frac{2 \pi i}{k}$ is a generator of the cyclic group of $k$ th roots of unity.

Let us observe that $\mathcal{K}_{\mathcal{G}}^{k}, k \geq 2$ are nonempty families. Indeed, the function $f=1$ belongs to $\mathcal{K}_{\mathcal{G}}^{k}$, because it satisfies the factorization (1.1) with $p=1 \in \mathcal{C}_{\mathcal{G}}$ and $h=1 \in \mathcal{M}_{\mathcal{G}}\left(\frac{k-1}{k)}\right)$.

In the future, we will use a characterization of the family $\mathcal{K}_{\mathcal{G}}^{k}$ by a notion of $(j, k)$ symmetry, which is connected with a functional decomposition with respect to the above group.

Let us observe that bounded complete $n$-circular domains $\mathcal{G}$ are $k$-symmetric sets for $k=2,3, \ldots$, that is $\varepsilon \mathcal{G}=\mathcal{G}$. For $j=0,1, \ldots, k-1$ we define the collections $\mathcal{F}_{j, k}(\mathcal{G})$ of functions $(j, k)$-symmetrical, i.e., all functions $f: \mathcal{G} \rightarrow \mathbb{C}$ such that

$$
f(\varepsilon z)=\varepsilon^{j} f(z), z \in \mathcal{G} .
$$

If $n=1$ and $\mathcal{G}=\mathcal{U}$, then we write $\mathcal{F}_{j, k}(\mathcal{U})$.

The mentioned functional decomposition appears in the following result from [14].

Theorem A For every function $f: \mathcal{G} \rightarrow \mathbb{C}$ there exists exactly one sequence of functions $f_{j, k} \in \mathcal{F}_{j, k}(\mathcal{G}), j=0,1, \ldots, k-1$, such that

$$
f=\sum_{j=0}^{k-1} f_{j, k}
$$

Moreover,

$$
f_{j, k}(z)=\frac{1}{k} \sum_{l=0}^{k-1} \varepsilon^{-j l} f\left(\varepsilon^{l} z\right), z \in \mathcal{G} .
$$

The functions $f_{j, k}$, which are uniquely determined by the above decomposition, will be called $(j, k)$-symmetrical components of the function $f$. Some interesting applications of the above partition may also be found in $[15,16]$ and [17]. 


\section{Results}

Now we can present a characterization of $f \in \mathcal{K}_{\mathcal{G}}^{k}$, simpler than (1.1).

Theorem 1 A function $f \in \mathcal{H}_{\mathcal{G}}(1)$ belongs to the family $\mathcal{K}_{\mathcal{G}}^{k}, k \geq 2$ if and only if there exists a function $g \in \mathcal{M}_{\mathcal{G}} \cap \mathcal{F}_{0, k}(\mathcal{G})$ and a function $p \in \mathcal{C}_{\mathcal{G}}$ such that

$$
\mathcal{L} f=p \cdot g \text {. }
$$

Proof Let $f \in \mathcal{K}_{\mathcal{G}}^{k}$. Then there exists $p \in \mathcal{C}_{\mathcal{G}}$ and $h \in \mathcal{M}_{\mathcal{G}}\left(\frac{k-1}{k}\right)$ such that

$$
\mathcal{L} f(z)=p(z) \cdot g(z), z \in \mathcal{G},
$$

where

$$
g(z)=\prod_{l=0}^{k-1} h\left(\varepsilon^{l} z\right), z \in \mathcal{G} .
$$

It is obvious that $g \in \mathcal{F}_{0, k}(\mathcal{G})$. We show that $g \in \mathcal{M}_{\mathcal{G}}$. To do it, using the differentiation product rule and the form of the operator $\mathcal{L}$, we have at $z \in \mathcal{G}$

$$
\frac{\mathcal{L} g(z)}{g(z)}=1+\frac{D g(z)(z)}{g(z)}=1+\sum_{l=0}^{k-1} \frac{D h\left(\varepsilon^{l} z\right)\left(\varepsilon^{l} z\right)}{h\left(\varepsilon^{l} z\right)}=1-k+\sum_{l=0}^{k-1} \frac{\mathcal{L} h\left(\varepsilon^{l} z\right)}{h\left(\varepsilon^{l} z\right)}
$$

Hence and by the fact that $h \in \mathcal{M}_{\mathcal{G}}\left(\frac{k-1}{k}\right)$, we obtain that $\operatorname{Re} \frac{\mathcal{L} g(z)}{g(z)}>1-k+k \frac{k-1}{k}=0$. Thus $g \in \mathcal{M}_{\mathcal{G}}$.

Now, let us suppose that $f$ satisfies the equality (2.1), with a $p \in \mathcal{C}_{\mathcal{G}}$ and a $g \in$ $\mathcal{M}_{\mathcal{G}} \cap \mathcal{F}_{0, k}(\mathcal{G})$. Let us put $h(z)=(g(z))^{\frac{1}{k}}, z \in \mathcal{G}$, with the power function taking the value 1 at the point 1 . Since $g(z) \neq 0$ (see [1]), the function $h$ is holomorphic. It remains to show that $h \in \mathcal{M}_{\mathcal{G}}\left(\frac{k-1}{k}\right)$ and the equality (1.1) is fulfilled. To this end we compute step by step

$$
\begin{aligned}
\operatorname{Re} \frac{\mathcal{L} h(z)}{h(z)} & =\operatorname{Re} \frac{\mathcal{L}(g(z))^{\frac{1}{k}}}{(g(z))^{\frac{1}{k}}}=1+\frac{1}{k} \operatorname{Re} \frac{(g(z))^{\frac{1}{k}-1} D g(z)(z)}{(g(z))^{\frac{1}{k}}} \\
& =1+\frac{1}{k} \operatorname{Re} \frac{D g(z)(z)}{g(z)}=\frac{k-1}{k}+\frac{1}{k} \operatorname{Re} \frac{\mathcal{L} g(z)}{g(z)}>\frac{k-1}{k} .
\end{aligned}
$$

The formula (1.1) follows from the definition of the function $h$. Indeed,

$$
g(z)=(h(z))^{k}=\prod_{l=0}^{k-1} h\left(\varepsilon^{l} z\right), \quad z \in \mathcal{G}
$$

because $h \in \mathcal{F}_{0, k}(\mathcal{G})$.

The proof is complete. 
Now we consider an extremal problem for $f \in \mathcal{K}_{\mathcal{G}}^{k}$. More precisely, we look for some estimates for $\mathcal{G}$-balances of $m$-homogeneous polynomias $Q_{f, m}$ of its unique power series expansion

$$
f(z)=1+\sum_{m=1}^{\infty} Q_{f, m}(z), z \in \mathcal{G} .
$$

In our considerations the Minkowski function

$$
\mu_{\mathcal{G}}(z)=\inf \left\{t>0: \frac{1}{t} z \in \mathcal{G}\right\}, z \in \mathbb{C}^{n},
$$

will be very useful. This function gives a possibility to redefine the domain $\mathcal{G}$ and its boundary $\partial \mathcal{G}$ as follows:

$$
\mathcal{G}=\left\{z \in \mathbb{C}^{n}: \mu_{\mathcal{G}}(z)<1\right\}, \partial \mathcal{G}=\left\{z \in \mathbb{C}^{n}: \mu_{\mathcal{G}}(z)=1\right\}
$$

The notion of $\mathcal{G}$-balance of $m$-homogeneous polynomial $Q_{m}: \mathbb{C}^{n} \rightarrow \mathbb{C}, m \in \mathbb{N} \cup\{0\}$, was defined in [3] as the quantity

$$
\mu_{\mathcal{G}}\left(Q_{m}\right)=\sup _{w \in \mathbb{C}^{n} \backslash\{0\}} \frac{\left|Q_{m}(w)\right|}{\left(\mu_{\mathcal{G}}(w)\right)^{m}}=\sup _{v \in \partial \mathcal{G}}\left|Q_{m}(v)\right|=\sup _{u \in \mathcal{G}}\left|Q_{m}(\mathcal{U})\right| .
$$

The $\mathcal{G}$-balance $\mu_{\mathcal{G}}\left(Q_{m}\right)$ generalizes the norm $\left\|Q_{m}\right\|$ of the polynomial $Q_{m}$ and if $\mathcal{G}$ is convex, then $\mu_{\mathcal{G}}\left(Q_{m}\right)$ reduces to $\left\|Q_{m}\right\|$, because

$$
\left|Q_{m}(w)\right| \leq \mu_{\mathcal{G}}\left(Q_{m}\right)\left(\mu_{\mathcal{G}}(w)\right)^{m}, w \in \mathbb{C}^{n}
$$

and for bounded convex complete $n$-circular domains $\mathcal{G}$ also $\mu_{\mathcal{G}}(w)=\|w\|$ (see, e.g., [21]).

We present the announced estimates of $\mathcal{G}$-balances $\mu_{\mathcal{G}}\left(Q_{f, m}\right)$ of $m$-homogeneous polynomials $Q_{f, m}$ from the Taylor series of $f \in \mathcal{M}_{\mathcal{G}}^{k}$ in the following theorem.

Theorem 2 If the expansion of the function $f \in \mathcal{K}_{\mathcal{G}}^{k}, k \geq 2$, into a series of $m$ homogenous polynomials $Q_{f, m}$ has the form (2.2), then for the $\mathcal{G}$-balances $\mu_{\mathcal{G}}\left(Q_{f, m}\right)$ of polynomials $Q_{f, m}$ the following sharp estimate hold:

$$
\mu_{\mathcal{G}}\left(Q_{f, m}\right) \leq\left\{\begin{array}{l}
\frac{2}{m} \prod_{p=1}^{\frac{m}{k}-1}\left(1+\frac{2}{p k}\right) \text { for } m=k, 2 k, 3 k, \ldots \\
\frac{2}{m+1} \prod_{p=1}^{\left\lfloor\frac{m}{k}\right\rfloor}\left(1+\frac{2}{p k}\right) \text { for remaining } m \in \mathbb{N}
\end{array},\right.
$$

where $\lfloor q\rfloor$ means the integral part of the number $q$. We use a standard convention that the product $\prod_{l=l_{1}}^{l_{2}} a_{l}$ is equal to 1 for $l_{2}<l_{1}$. 
Proof Let $f \in \mathcal{K}_{\mathcal{G}}^{k}$ be arbitrarily fixed. Then, by Theorem 1, the factorization (2.1) holds with a function $p \in \mathcal{C}_{\mathcal{G}}$ of the form

$$
p(z)=1+\sum_{\nu=1}^{\infty} Q_{p, v}(z), z \in \mathcal{G}
$$

and a function $g \in \mathcal{M}_{\mathcal{G}} \cap \mathcal{F}_{0, k}(\mathcal{G})$ of the form

$$
g(z)=1+\sum_{\nu=1}^{\infty} Q_{g, k \nu}(z), \quad z \in \mathcal{G} .
$$

From the above, by the series expansion of $\mathcal{L} f$

$$
\mathcal{L} f(z)=1+\sum_{m=1}^{\infty} Q_{\mathcal{L} f, m}(z)=1+\sum_{m=1}^{\infty}(m+1) Q_{f, m}(z), \quad z \in \mathcal{G}
$$

and by the equalities $Q_{f, 0}=Q_{p, 0}=Q_{g, 0}=1$, we obtain the recursive formula for $m \in \mathbb{N}$

$$
(m+1) Q_{f, m}(z)=\sum_{l=0}^{\left\lfloor\frac{m}{k}\right\rfloor} Q_{g, k l}(z) Q_{p, m-k l}(z), \quad z \in \mathcal{G} .
$$

Hence

$$
(m+1)\left|Q_{f, m}(z)\right| \leq \sum_{l=0}^{\left\lfloor\frac{m}{k}\right\rfloor}\left|Q_{g, k l}(z)\right|\left|Q_{p, m-k l}(z)\right|, \quad z \in \mathcal{G} .
$$

Since

$$
\left|Q_{p, v}(z)\right| \leq 2, \quad v \in \mathbb{N}, \quad z \in \mathcal{G}
$$

(see [1]) we need some bounds for $\left|Q_{g, k \mu}(z)\right|$. We show that for $g \in \mathcal{M}_{\mathcal{G}} \cap \mathcal{F}_{0, k}(\mathcal{G})$ and $\mu \in \mathbb{N}$ there hold the inequalities

$$
\left|Q_{g, k \mu}(z)\right| \leq \frac{2}{k \mu} \prod_{\nu=1}^{\mu-1}\left(1+\frac{2}{k v}\right), z \in \mathcal{G}
$$

For this purpose let us observe that for each $z \in \mathcal{G}$, the function

$$
G(\zeta)=\zeta g(\zeta z), \zeta \in \mathcal{U}
$$

belongs to the family $\mathcal{S}^{*} \cap \mathcal{F}_{1, k}(\mathcal{U})$ of $(1, k)$-symmetric univalent starlike mappings (in the unit $\operatorname{disc} \mathcal{U}$ ) and its Taylor series has the form

$$
G(\zeta)=\zeta+\sum_{\mu=1}^{\infty} b_{k \mu+1} \zeta^{k \mu+1}=1+\sum_{\mu=1}^{\infty} Q_{g, k \mu}(z) \zeta^{k \mu+1}, \zeta \in \mathcal{U}
$$


Thus, in view of the estimates [25] of the coefficients of functions from $\mathcal{S}^{*} \cap \mathcal{F}_{1, k}(\mathcal{U})$ we get the announced bounds (2.6).

In two next parts of the proof we use also the fact [4] that for every $k, s \in \mathbb{N} \backslash\{1\}$ there holds the identity:

$$
1+\frac{2}{k}+\sum_{l=2}^{s} \frac{2}{l k} \prod_{\nu=1}^{l-1}\left(1+\frac{2}{\nu k}\right)=\prod_{\nu=1}^{s}\left(1+\frac{2}{\nu k}\right) .
$$

Now, we will estimate the quantities $\left|Q_{f, m}(z)\right|, z \in \mathcal{G}$, using all the conditions (2.4)(2.7).

First let us assume that $m=k s$, where $s \in \mathbb{N}$. Since $Q_{p, m-k l}(z)=1$ for $l=s$, we get from (2.4) that

$$
(m+1)\left|Q_{f, m}(z)\right| \leq\left|Q_{g, k s}(z)\right|+2 \sum_{l=0}^{s-1}\left|Q_{g, k l}(z)\right|, z \in \mathcal{G} .
$$

Thus for $z \in \mathcal{G}$, in view of (2.6) and (2.7),

$$
\begin{aligned}
(m+1)\left|Q_{f, m}(z)\right| & \leq \frac{2}{s k} \prod_{\nu=1}^{s-1}\left(1+\frac{2}{v k}\right)+2\left[1+\frac{2}{k}+\sum_{l=2}^{s-1} \frac{2}{l k} \prod_{\nu=1}^{l-1}\left(1+\frac{2}{v k}\right)\right] \\
& =\frac{-2}{s k} \prod_{\nu=1}^{s-1}\left(1+\frac{2}{v k}\right)+2\left[1+\frac{2}{k}+\sum_{l=2}^{s} \frac{2}{l k} \prod_{\nu=1}^{l-1}\left(1+\frac{2}{v k}\right)\right] \\
& \leq \frac{-2}{s k} \prod_{\nu=1}^{s-1}\left(1+\frac{2}{v k}\right)+2 \prod_{\nu=1}^{s}\left(1+\frac{2}{v k}\right) \\
& =2 \frac{(s k+1)}{s k} \prod_{\nu=1}^{s-1}\left(1+\frac{2}{v k}\right) .
\end{aligned}
$$

Hence, for $m=k, 2 k, 3 k, \ldots$

$$
\left|Q_{f, m}(z)\right| \leq \frac{2}{m} \prod_{\nu=1}^{\frac{m}{k}-1}\left(1+\frac{2}{\nu k}\right), z \in \mathcal{G}
$$

Now let us consider the case $m=k s+r$, where $s \in \mathbb{N} \cup\{0\}$ and $r \in\{1,2, \ldots, k-1\}$. In this case we apply in (2.4) the inequality $\left|Q_{p, m-k l}(z)\right| \leq 2, l=0, \ldots, s=\left\lfloor\frac{m}{k}\right\rfloor$, which follows from estimates (2.5), because $m-k l>0$. Thus, in view of (2.6) and (2.7) we get step by step 


$$
\begin{aligned}
(m+1)\left|Q_{f, m}(z)\right| & \leq 2 \sum_{l=0}^{\left\lfloor\frac{m}{k}\right\rfloor}\left|Q_{g, k l}(z)\right| \leq 2\left[1+\frac{2}{k}+\sum_{l=2}^{\left\lfloor\frac{m}{k}\right\rfloor} \frac{2}{l k} \prod_{\nu=1}^{l-1}\left(1+\frac{2}{v k}\right)\right] \\
& \leq 2 \prod_{\nu=1}^{\left\lfloor\frac{m}{k}\right\rfloor}\left(1+\frac{2}{\nu k}\right) .
\end{aligned}
$$

Summing up the results of both cases we get

$$
\left|Q_{f, m}(z)\right| \leq\left\{\begin{array}{l}
\frac{2}{m} \prod_{\nu=1}^{\frac{m}{k}-1}\left(1+\frac{2}{v k}\right) \text { for } m=k, 2 k, 3 k, \ldots \\
\frac{2}{m+1} \prod_{\nu=1}^{\left\lfloor\frac{m}{k}\right\rfloor}\left(1+\frac{2}{v k}\right) \text { for remaining } m \in \mathbb{N}
\end{array}, z \in \mathcal{G}\right.
$$

and consequently

$$
\sup _{z \in \mathcal{G}}\left|Q_{f, m}(z)\right| \leq\left\{\begin{array}{l}
\frac{2}{m} \prod_{\nu=1}^{\frac{m}{k}-1}\left(1+\frac{2}{\nu k}\right) \text { for } m=k, 2 k, 3 k, \ldots \\
\frac{2}{m+1} \prod_{\nu=1}^{\left\lfloor\frac{m}{k}\right\rfloor}\left(1+\frac{2}{\nu k}\right) \text { for remaining } m \in \mathbb{N}
\end{array} .\right.
$$

These inequalities and the definition of $\mathcal{G}$-balances $\mu_{\mathcal{G}}\left(Q_{f, m}\right)$ of $m$-homogeneous polynomials imply the estimates from the statement of the theorem.

Now, we will show the sharpness of the above estimates.

For the linear functional $I=\left(\mu_{\mathcal{G}}(J)\right)^{-1} J$, with

$$
J(z)=\sum_{l=1}^{n} z_{l}, z=\left(z_{1}, \ldots, z_{n}\right) \in \mathbb{C}^{n},
$$

let us denote by $\mathcal{Z}$ an analytic set $\mathcal{G} \cap I^{-1}\{0\}$ and let $I^{m}(z)=(I z)^{m}, z \in \mathcal{G}, m \in$ $\mathbb{N} \cup\{0\}$. The equalities in our estimates are achieved for the following function $f \in$ $\mathcal{K}_{\mathcal{G}}^{k}, k \geq 2$,

$$
f(z)=\left\{\begin{array}{cc}
\frac{\sum_{l=0}^{k-1} I^{l-1}(z)}{\left(1-I^{k}(z)\right)^{\frac{2}{k}}}-\frac{1}{I(z)}-\sum_{l=3}^{k-1} \frac{l-2}{l} I^{l-1}(z) H\left(\frac{2}{k}, \frac{l}{k}, \frac{l+k}{k}, I^{k}(z)\right) \text { for } z \in \mathcal{G} \backslash \mathcal{Z}, \\
1 \quad \text { for } z \in \mathcal{Z}
\end{array}\right.
$$

where $H(a, b, c, \zeta): \mathcal{U} \rightarrow \mathbb{C}$ is a hypergeometric function

$$
H(a, b, c, \zeta)=\sum_{\nu=0}^{\infty} \frac{(a)_{v}(b)_{v}}{(c)_{v}} \frac{\zeta^{v}}{\nu !}, \zeta \in \mathcal{U}
$$


defined by Pochhamer symbols $(a)_{v},(b)_{v},(c)_{v}$ :

$$
(a)_{v}=\left\{\begin{array}{ll}
a(a+1) \ldots(a+v-1), & v \in \mathbb{N} \\
1, & v=0
\end{array},\right.
$$

and the branch of the power function $x^{\frac{2}{k}}$ takes the value 1 at the point $x=1$. In the case $k=2,3$ we use a standard convention that the sum

$$
\sum_{l=3}^{k-1} \frac{l-2}{l} I^{l-1}(z) H\left(\frac{2}{k}, \frac{l}{k}, \frac{l+k}{k}, I^{k}(z)\right), z \in \mathcal{G}
$$

is equal to zero, if the superscript of the sum is smaller than the subscript.

In the paper [4], it was proven that the above function gives the equalities in the bounds from the statement of the theorem. It remains to show that $f \in \mathcal{K}_{\mathcal{G}}^{k}$ for $k \geq 2$. To do it, let us observe that as shown in [4]

$$
\mathcal{L} f(z)=\frac{1+I(z)}{1-I(z)} \frac{1}{\left(1-I^{k}(z)\right)^{\frac{2}{k}}}, z \in \mathcal{G} .
$$

This implies, in view of Theorem 1 , the relation $f \in \mathcal{K}_{\mathcal{G}}^{k}$, because the functions

$$
p(z)=\frac{1+I(z)}{1-I(z)}, g(z)=\frac{1}{\left(1-I^{k}(z)\right)^{\frac{2}{k}}}, z \in \mathcal{G}
$$

belong to $\mathcal{C}_{\mathcal{G}}$ and to $\mathcal{M}_{\mathcal{G}} \cap \mathcal{F}_{0, k}(\mathcal{G})$, respectively.

We use the estimates of $\mathcal{G}$-balances $\mu_{\mathcal{G}}\left(Q_{f, m}\right)$ of polynomials $Q_{f, m}$ to solve the mentioned separation problem for the families $\mathcal{V}_{\mathcal{G}}, \mathcal{K}_{\mathcal{G}}^{k}, \mathcal{R}_{\mathcal{G}}$. We prove the following theorem:

Theorem 3 For every $k \geq 2$ there holds the double inclusion

$$
\mathcal{V}_{\mathcal{G}} \varsubsetneqq \mathcal{K}_{\mathcal{G}}^{k} \varsubsetneqq \mathcal{R}_{\mathcal{G}}
$$

Proof We start with the inclusion $\mathcal{V}_{\mathcal{G}} \subset \mathcal{K}_{\mathcal{G}}^{k}$. To do it, let us assume that $f \in \mathcal{V}_{\mathcal{G}}$, then $\mathcal{L} f \in \mathcal{C}_{\mathcal{G}}$. Putting $p=\mathcal{L} f$ and $h=1$, we obtain the factorization (1.1) with $p \in \mathcal{C}_{\mathcal{G}}$ and $g=1 \in \mathcal{M}_{\mathcal{G}} \cap \mathcal{F}_{0, k}(\mathcal{G})$. Hence $f \in \mathcal{K}_{\mathcal{G}}^{k}$. It remains to show the relation $\mathcal{V}_{\mathcal{G}} \neq \mathcal{K}_{\mathcal{G}}^{k}$. To do it, let us observe that for $f \in \mathcal{V}_{\mathcal{G}}$ there hold the sharp estimates $\mu_{\mathcal{G}}\left(Q_{f, m}\right) \leq$ $\frac{2}{m+1}, m \in \mathbb{N}$ (cf., eg., [1]), while for $f \in \mathcal{K}_{\mathcal{G}}^{k}$ the sharp estimates $\mu_{\mathcal{G}}\left(Q_{f, m}\right) \leq$ $B(m)$ (Theorem 2.), with the obvious bound $B(m)>\frac{2}{m+1}, m \in \mathbb{N} \backslash\{1\}$. Hence, the extremal function $f \in \mathcal{K}_{\mathcal{G}}^{k}$ does not belong to $\mathcal{V}_{\mathcal{G}}$.

Now we prove that $\mathcal{K}_{\mathcal{G}}^{k} \subset \mathcal{R}_{\mathcal{G}}$. To this end, let us suppose that $f \in \mathcal{K}_{\mathcal{G}}^{k}$. Then there exist functions $p \in \mathcal{C}_{\mathcal{G}}, g \in \mathcal{M}_{\mathcal{G}} \cap \mathcal{F}_{0, k}(\mathcal{G})$ such that $\mathcal{L} f=p \cdot g$. Denoting $\varphi=\mathcal{L}^{-1} g$, we have that $\varphi \in \mathcal{N}_{\mathcal{G}}$ (by the Aleksander type theorem [1]) and $\mathcal{L} f=p \mathcal{L} \varphi$. Thus 
$f \in \mathcal{R}_{\mathcal{G}}$. It remains to show the relation $\mathcal{K}_{\mathcal{G}}^{k} \neq \mathcal{R}_{\mathcal{G}}$. For this purpose, let us observe that in the above estimates $\mu_{\mathcal{G}}\left(Q_{f, m}\right) \leq B(m), m \in \mathbb{N}$, we have $B(m) \leq 1, m \in \mathbb{N}$ (see below), while for $f \in \mathcal{R}_{\mathcal{G}}$ there hold the sharp estimates $\mu_{\mathcal{G}}\left(Q_{f, m}\right) \leq m+1$ (see for instance [1]). Therefore, the extremal function $f \in \mathcal{R}_{\mathcal{G}}$ does not belong to $\mathcal{K}_{\mathcal{G}}^{k}$.

To complete the proof, we show that $B(m) \leq 1, m \in \mathbb{N}$. To do it, we consider two cases, according to the partition $m=k s+r, r \in\{0,1, \ldots, k-1\}$, from the proof of Theorem 2.

1. Let us suppose that $r=0$. Then, if $s=\frac{m}{k}=1$, we see that the superscript $s-1$ of the first product in Theorem 2 is smaller than its subscript 1 . Hence, we replace the referred product by 1 and consequently, we get $\mu_{\mathcal{G}}\left(Q_{f, m}\right) \leq \frac{2}{m} \leq 1$, because $m=k \geq 2$. Next, if $s \geq 2$, then from Theorem 2 , by the inequality $1+\frac{2}{v k} \leq \frac{v+1}{v}, v \in$ $\mathbb{N}, k \in \mathbb{N} \backslash\{1\}$, we obtain

$$
\mu_{\mathcal{G}}\left(Q_{f, m}\right) \leq \frac{2}{m} \prod_{\nu=1}^{s-1} \frac{v+1}{v} \leq \frac{2}{m} s=\frac{2}{k} \leq 1
$$

2. Let us suppose that $r \in\{1, \ldots, k-1\}$. Then, if $s=\left\lfloor\frac{m}{k}\right\rfloor=0$, we see that the superscript of the second product in Theorem 2 is smaller than its subscript. Hence we replace the referred product by 1 and consequently, we get $\mu_{G}\left(Q_{f, m}\right) \leq \frac{2}{m+1} \leq 1$, because $m \leq k-1$. Next, if $s=\left\lfloor\frac{m}{k}\right\rfloor \geq 1$, then similarly as in step 1 , we obtain

$$
\mu_{\mathcal{G}}\left(Q_{f, m}\right) \leq \frac{2}{m+1} \prod_{\nu=1}^{s} \frac{v+1}{v} \leq \frac{2}{m+1}(s+1) \leq \frac{2(s+1)}{k s+2} \leq \frac{2(s+1)}{2 s+2} \leq 1 .
$$

Now, we give a growth theorem for $f \in \mathcal{K}_{\mathcal{G}}^{k}$ and its Temljakov transform $\mathcal{L} f$.

Theorem 4 For functions $f \in \mathcal{K}_{\mathcal{G}}^{k}$ there follow the following sharp estimates

$$
\begin{aligned}
\frac{1-r}{1+r} \frac{1}{\left(1+r^{k}\right)^{\frac{2}{k}}} & \leq|\mathcal{L} f(z)| \leq \frac{1+r}{1-r} \frac{1}{\left(1-r^{k}\right)^{\frac{2}{k}}}, r=\mu_{\mathcal{G}}(z) \in[0,1), \\
\frac{1}{r} \int_{0}^{r} \frac{1-\varrho}{1+\varrho} \frac{1}{\left(1+\varrho^{k}\right)^{\frac{2}{k}}} d \varrho & \leq|f(z)| \leq \frac{1}{r} \int_{0}^{r} \frac{1+\varrho}{1-\varrho} \frac{1}{\left(1-\varrho^{k}\right)^{\frac{2}{k}}} d \varrho \\
r & =\mu_{\mathcal{G}}(z) \in[0,1) .
\end{aligned}
$$

Proof First, let us observe that the above estimates are true for $z=0$ (in (2.10) the values at $r=0$, of the left and right hand sides, mean the limit if $r \rightarrow 0^{+}$). Thus, in the sequel we will assume that $z \in \mathcal{G} \backslash\{0\}$. We start with the estimates (2.9). Since $f \in \mathcal{K}_{\mathcal{G}}^{k}$, there exist a function $p \in \mathcal{C}_{\mathcal{G}}$ and a function $g \in \mathcal{M}_{\mathcal{G}} \cap \mathcal{F}_{0, k}(\mathcal{G})$ such that the factorization (2.1) holds. Therefore, we show for such functions $g$ the following inequalities 


$$
\frac{1}{\left(1+r^{k}\right)^{\frac{2}{k}}} \leq|g(z)| \leq \frac{1}{\left(1-r^{k}\right)^{\frac{2}{k}}}, r=\mu_{\mathcal{G}}(z) \in(0,1) .
$$

To this aim, let us fix arbitrarily a point $z \in \mathcal{G}$ such that $\mu_{\mathcal{G}}(z)=r \in(0,1)$ and let us consider the function

$$
G(\zeta)=\zeta g\left(\zeta \frac{z}{\mu_{\mathcal{G}}(z)}\right), \zeta \in \mathcal{U}
$$

Then $G$ is $(1, k)$-symmetric, holomorphic, normalized and satisfies the condition

$$
\operatorname{Re} \frac{\zeta G^{\prime}(\zeta)}{G(\zeta)}=\operatorname{Re} \frac{\mathcal{L} g\left(\zeta \frac{z}{\mu_{\mathcal{G}}(z)}\right)}{g\left(\zeta \frac{z}{\mu_{\mathcal{G}}(z)}\right)}>0, \zeta \in \mathcal{U}
$$

Hence $G \in \mathcal{S}^{*} \cap \mathcal{F}_{1, k}(\mathcal{U})$ and by [9, Thm. 2.2.13]

$$
\frac{|\zeta|}{\left(1+|\zeta|^{k}\right)^{\frac{2}{k}}} \leq|G(\zeta)| \leq \frac{|\zeta|}{\left(1-|\zeta|^{k}\right)^{\frac{2}{k}}}, \zeta \in \mathcal{U}
$$

Putting $\zeta=\mu_{\mathcal{G}}(z)$ in the above we obtain, by the definition of the function $G$, the announced inequality.

On the other hand, there hold for $p \in \mathcal{C}_{\mathcal{G}}$ the following estimates [1]

$$
\frac{1-r}{1+r} \leq|p(z)| \leq \frac{1+r}{1-r}, r=\mu_{\mathcal{G}}(z) \in(0,1),
$$

Using the estimates of $|p(z)|$ and $|g(z)|$ we get the estimates (2.9). The sharpness of the upper bounds (2.9) confirms the function given by (2.8). Indeed, for $r \in(0,1)$ and function $f \in \mathcal{K}_{\mathcal{G}}^{k}$ given by (2.8), we get

$$
\mathcal{L} f(z)=\frac{1+r}{1-r} \frac{1}{\left(1-r^{k}\right)^{\frac{2}{k}}}
$$

at points $z \in \mathcal{G}, \mu_{\mathcal{G}}(z)=r \in(0,1)$ such that $I(z)=r$ (this condition is fulfilled by the points $z=r z^{*}$, where $z^{*} \in \partial \mathcal{G}$ and $\left.I\left(z^{*}\right)=1\right)$.

The sharpness of the lower bounds (2.9) can be proven in a similar way.

Now, we prove the estimates (2.10). To obtain the upper bound (2.10), we use the proved above upper bound (2.9) and the fact that the Temljakov operator $\mathcal{L}$ is invertible and

$$
\mathcal{L}^{-1} u(z)=\int_{0}^{1} u(t z) d t, u \in \mathcal{H}_{\mathcal{G}}, z \in \mathcal{G} .
$$


Indeed, we have for $f \in \mathcal{K}_{\mathcal{G}}^{k}$ and $z \in \mathcal{G}, \mu_{\mathcal{G}}(z)=r \in(0,1)$,

$$
\begin{aligned}
|f(z)| & =\left|\mathcal{L}^{-1} \mathcal{L} f(z)\right|=\left|\int_{0}^{1} \mathcal{L}(t z) d t\right| \leq \int_{0}^{1} \frac{1+r t}{(1-r t)\left(1-(r t)^{k}\right)^{\frac{2}{k}}} d t \\
& =\frac{1}{r} \int_{0}^{r} \frac{1+\varrho}{(1-\varrho)\left(1-\varrho^{k}\right)^{\frac{2}{k}}} d \varrho .
\end{aligned}
$$

To prove the lower bound (2.10) let us consider the function

$$
F(\zeta)=\zeta f\left(\zeta \frac{z}{\mu_{\mathcal{G}}(z)}\right), \zeta \in \mathcal{U}
$$

with arbitrarily fixed $f \in \mathcal{K}_{\mathcal{G}}^{k}$ and $z \in \mathcal{G}, \mu_{\mathcal{G}}(z)=r \in(0,1)$. Since

$$
F^{\prime}(\zeta)=\mathcal{L} f\left(\zeta \frac{z}{\mu_{\mathcal{G}}(z)}\right), \zeta \in \mathcal{U}
$$

we get, by Theorem 1, that there exist functions $g \in \mathcal{M}_{\mathcal{G}} \cap \mathcal{F}_{0, k}(\mathcal{G})$ and $p \in \mathcal{C}_{\mathcal{G}}$ such that the factorization (2.1) is true. Thus

$$
F^{\prime}(\zeta)=P(\zeta) \cdot G(\zeta), \zeta \in \mathcal{U}
$$

where for $\zeta \in \mathcal{U}$

$$
G(\zeta)=\zeta g\left(\zeta \frac{z}{\mu_{\mathcal{G}}(z)}\right), P(\zeta)=p\left(\zeta \frac{z}{\mu_{\mathcal{G}}(z)}\right)
$$

Moreover, $G \in \mathcal{S}^{*} \cap \mathcal{F}_{1, k}(\mathcal{U})$ ( see the proof of the estimates (2.9)) and $P: \mathcal{U} \rightarrow$ $\mathbb{C}, P(0)=1$, is a holomorphic function with a positive real part. Therefore, $F$ belongs to a subclass $\mathcal{K}^{(k)}$ (considered in [22] and for $k=2$ in [8]) of the class of close-to-convex functions. Hence, $F$ is univalent in the $\operatorname{disc} \mathcal{U}$.

On the other hand, by the lower bound (2.9), we have that

$$
\left|F^{\prime}(\zeta)\right| \geq \frac{1-|\zeta|}{1+|\zeta|} \frac{1}{\left(1+|\zeta|^{k}\right)^{\frac{2}{k}}}
$$

because $r=\mu_{\mathcal{G}}\left(\zeta \frac{z}{\mu_{\mathcal{G}}(z)}\right)=|\zeta|$. Now we show that

$$
|F(\zeta)| \geq \int_{0}^{r} \frac{1-\varrho}{1+\varrho} \frac{1}{\left(1+\varrho^{k}\right)^{\frac{2}{k}}} d \varrho,|\zeta|=r \in(0,1)
$$


To this aim, it is sufficient to show that it holds for the nearest point $F\left(\zeta_{0}\right)$ from zero $\left(\left|\zeta_{0}\right|=r \in(0,1)\right)$, otherwise, we have $|F(\zeta)| \geq\left|F\left(\zeta_{0}\right)\right|,|\zeta|=r$. Since $F$ is univalent in the $\operatorname{disc} \mathcal{U}$, the original image of the line segment $\overline{0, F\left(\zeta_{0}\right)}$ is a piece of $\operatorname{arc} F^{-1}\left(\overline{0, F\left(\zeta_{0}\right)}\right)$ in the disc $r \overline{\mathcal{U}}$. Thus

$$
\begin{aligned}
\left|F\left(\zeta_{0}\right)\right| & =\int_{0, F\left(\zeta_{0}\right)}|d w|=\int_{F^{-1}\left(\overline{0, F\left(\zeta_{0}\right)}\right)}\left|F^{\prime}(\zeta)\right| \mid d \zeta \\
& \left.\geq \int_{0}^{r} \frac{1-\varrho}{1+\varrho} \frac{1}{\left(1+\varrho^{k}\right)^{\frac{2}{k}}} d \varrho, r \in(0,1)\right) .
\end{aligned}
$$

Thus, by the definition of $F$, we get

$$
\left|\zeta f\left(\zeta \frac{z}{\mu_{\mathcal{G}}(z)}\right)\right| \geq \int_{0}^{r} \frac{1-\varrho}{1+\varrho} \frac{1}{\left(1+\varrho^{k}\right)^{\frac{2}{k}}} d \varrho,|\zeta|=r \in(0,1) .
$$

Hence, putting $\zeta=\mu_{\mathcal{G}}(z)=r \in(0,1)$, we have the lower bound (2.10).

Finally, let us note that we obtain the equalities in the inequalities (2.10) for the function (2.8) in adequate points $z \in \mathcal{G}$.

We close the paper with a sufficient condition guaranteeing that a function $f \in$ $\mathcal{H}_{\mathcal{G}}(1)$ belongs to $\mathcal{K}_{\mathcal{G}}^{k}$. We formulate it in the term of $\mathcal{G}$-balances of $m$-honogeous polynomials in developments of functions from $\mathcal{H}_{\mathcal{G}}(1)$.

Theorem 5 Let $f \in \mathcal{H}_{\mathcal{G}}(1)$ has the form (2.2). If there exists a function $g \in \mathcal{M}_{\mathcal{G}} \cap$ $\mathcal{F}_{0, k}(\mathcal{G})$ of the form (2.3) such that

$$
\sum_{m=1}^{\infty}(m+1) \mu_{\mathcal{G}}\left(Q_{f, m}\right)+\sum_{m=1}^{\infty} \mu_{\mathcal{G}}\left(Q_{g, m k}\right) \leq 1
$$

then $f \in \mathcal{K}_{\mathcal{G}}^{k}$.

Proof Since $g$, as a function from $\mathcal{M}_{\mathcal{G}}$ omits zero [1], we will prove that

$$
\operatorname{Re} \frac{\mathcal{L} f(z)}{g(z)}>0, z \in \mathcal{G} \text {. }
$$

To do it, we compute step by step 


$$
\begin{array}{rl}
\mid \mathcal{L} & f(z)-g(z)|-| \mathcal{L} f(z)+g(z) \mid \\
= & \left|\sum_{m=1}^{\infty}(m+1) Q_{f, m}(z)-\sum_{m=1}^{\infty} Q_{g, m k}(z)\right|-\mid 2+\sum_{m=1}^{\infty}(m+1) Q_{f, m}(z) \\
& +\sum_{m=1}^{\infty} Q_{g, m k}(z) \mid \\
\leq & 2\left[\sum_{m=1}^{\infty}(m+1)\left|Q_{g, m k}(z)\right|+\sum_{m=1}^{\infty}\left|Q_{g, m k}(z)\right|-1\right] \\
\leq & 2\left[\sum_{m=1}^{\infty}(m+1) \mu_{\mathcal{G}}\left(Q_{f, m}\right)+\sum_{m=1}^{\infty} \mu_{\mathcal{G}}\left(Q_{g, m k}\right)-1\right] \leq 0
\end{array}
$$

Thus

$$
\left|\frac{\mathcal{L} f(z)}{g(z)}-1\right| \leq\left|\frac{\mathcal{L} f(z)}{g(z)}+1\right|, z \in \mathcal{G}
$$

and hence

$$
\operatorname{Re} \frac{\mathcal{L} f(z)}{g(z)} \geq 0, z \in \mathcal{G} .
$$

This gives the mentioned inequality by a maximum principle for pluriharmonic functions of several complex variables. Putting $p(z)=\frac{\mathcal{L} f(z)}{g(z)}, z \in \mathcal{G}$, we obtain that the transform $\mathcal{L} f$ has the factorization (1) with $g \underset{\mathcal{G}}{\in} \cap \mathcal{M}_{0, k}(\mathcal{G})$ and $p \in \mathcal{C}_{\mathcal{G}}$. Consequently, $f \in \mathcal{K}_{\mathcal{G}}^{k}$.

Open Access This article is distributed under the terms of the Creative Commons Attribution 4.0 International License (http://creativecommons.org/licenses/by/4.0/), which permits unrestricted use, distribution, and reproduction in any medium, provided you give appropriate credit to the original author(s) and the source, provide a link to the Creative Commons license, and indicate if changes were made.

\section{References}

1. Bavrin, I.I.: A class of regular bounded functions in the case of several complex variables and extreme problems in that class. Moskov Obl. Ped. Inst. Moskov (1976) (in Russian)

2. Długosz, R.: Embedding theorems for holomorphic functions of several complex variables. J. Appl. Anal. 19, 153-165 (2013)

3. Długosz, R., Leś, E.: Embedding theorems and extreme problems for holomorphic functions on circular domains of $\mathbb{C}^{n}$. Complex Var. Elliptic Equ. 59, 883-899 (2014)

4. Długosz, R., Liczberski, P.: An application of hypergeometric functions to a construction in several complex variables. J. Anal. Math. (2017) accepted for publication

5. Dobrowolska, K., Liczberski, P.: On some differential inequalities for holomorphic functions of many variables. Demonstr. Math. 14, 383-398 (1981)

6. Dziubiński, I., Sitarski, R.: On classes of holomorphic functions of many variables starlike and convex on some hypersurfaces. Demonstr. Math. 13, 619-632 (1980)

7. Fukui, S.: On the estimates of coefficients of analytic functions. Sci. Rep. Tokyo Kyoiku Daigaku Sect. A 10, 216-218 (1969)

8. Gao, C., Zhou, S.: On a class of analytic functions related to the starlike functions. Kyungpook Math. J. 45, 123-130 (2005) 
9. Graham, I., Kohr, G.: Geometric Function Theory in One and Higher Dimensions. Marcel Dekker Inc, New York (2003)

10. Hamada, H., Honda, T., Kohr, G.: Parabolic starlike mappings in several complex variables. Manuscr. Math. 123, 301-324 (2007)

11. Higuchi, T.: On coefficients of holomorphic functions of several complex variables. Sci. Rep. Tokyo Kyoiku Daigaku 8, 251-258 (1965)

12. Leś-Bomba, E., Liczberski, P.: On some family of holomorphic functions of several complex variables. Sci. Bull. Chelm Sect. Math. Comput. Sci. 2, 7-16 (2007)

13. Liczberski, P.: On the subordination of holomorphic mappings in $\mathbb{C}^{n}$. Demonstr. Math. 2, 293-301 (1986)

14. Liczberski, P., Połubiński, J.: On $(j, k)$-symmetrical functions. Math. Bohem. 120, 13-28 (1995)

15. Liczberski, P., Połubiński, J.: Functions $(j, k)$-symmetrical and functional equations with iterates of the unknown function. Publ. Math. Debr. 60, 291-305 (2002)

16. Liczberski, P., Połubiński, J.: Symmetrical series expansion of complex valued functions. N. Z. J. Math. 27, 245-253 (1998)

17. Liczberski, P., Połubiński, J.: A uniqueness theorem of Cartan-Gutzmer type for holomorphic mappings in $\mathbb{C}^{n}$. Ann. Pol. Math. 79, 121-127 (2002)

18. Marchlewska, A.: On a generalization of close-to-convexity for complex holomorphic functions in $\mathbb{C}^{n}$, . Demonstr. Math. 4, 847-856 (2005)

19. Michiwaki, Y.: Note on some coefficients in a starlike functions of two complex variables. Res. Rep. Nagaoka Tech. Coll. 1, 151-153 (1963)

20. Pfaltzgraff, J.A., Suffridge, T.J.: An extension theorem and linear invariant families generated by starlike maps. Ann. Univ. Mariae Curie Sklodowska Sect. A 53, 193-207 (1999)

21. Rudin, W.: Functional Analysis. McGraw-Hill Inc, New York (1991)

22. Seker, B.: On certain new subclass of close-to-convex functions. Appl. Math. Comput. 218, 1041-1045 (2011)

23. Stankiewicz, J.: Functions of two complex variables regular in halfspace. Folia Sci. Univ. Techn. Rzeszów. Math. 19, 107-116 (1996)

24. Temljakov, A.: Integral representation of functions of two complex variables. Izv. Ross. Akad. Nauk. Ser. Mat. 21, 89-92 (1957)

25. Waadeland, H.: Über k-fach symmetrische sternfö rmige schlichte Abbildungen des Einheitkreises. Math. Scand. 3, 150-154 (1955) 